\title{
Modified Distributed Energy Efficient Clustering Routing Protocol for Wireless Sensor Networks
}

\author{
Jeevan L J Pinto \\ Department of MCA \\ Srinivas Institute of PG Studies \\ Mangalore, Karnataka
}

\author{
Manjaiah D. H, Ph.D \\ Department of Computer Science \\ Mangalore University \\ Mangalore, Karnataka
}

\begin{abstract}
A Wireless Sensor Network (WSN) consists of thousands of sensor nodes with limited energy, memory and computational capability. Routing protocol with energy efficiency has been a challenging issue in the design of Wireless Sensor Networks. Various routing protocols are designed for transmission in WSNs. In this paper a mechanism has been proposed of designing a routing protocol by considering the initial and residual energy levels of the sensor nodes. The proposed routing protocol for wireless Sensor Networks enhances stability period, network life time and throughput quite significantly.
\end{abstract}

\section{Keywords}

Wireless Sensor Network (WSN), LEACH, Throughput, Energy Efficiency, Network Life Time

\section{INTRODUCTION}

Wireless Sensor Networks composed of thousands of sensor nodes which sense the physical environment in terms of temperature, light, vibration, sound etc. Wireless Sensor Networks enable us to use these small sensor nodes for applications like military applications, area monitoring, manufacturing etc. Since sensor nodes are power constrained devices, frequent and long-distance transmissions should be kept to minimum in order to prolong the network lifetime. Therefore the direct communication between nodes and the base station are not encouraged. The effective mechanism is to divide the network into several clusters, each electing one node as its cluster head. The elected cluster head collects the data from sensor nodes in the cluster which will be fused and then transmitted to the base station. The advantage of this is that only few nodes are required to transmit data over a long distance and then remaining nodes need only to do short distance transmission. As a result of this, more energy is saved and overall network life time can be prolonged.

On the basis of energy distribution among sensor nodes, WSNs are classified into homogenous and heterogeneous networks. Many energy-efficient routing protocols are designed based on the clustering where cluster-heads are elected periodically. Even-though the nodes are equipped with the same energy at the beginning, the networks cannot evolve equably for each node in expending energy due to the radio communication characteristics, random events such as shortterm link failure etc. Because of this, WSN are more possibly heterogeneous networks than homogeneous type. The algorithms such as Low Energy Adaptive Clustering Hierarchy (LEACH) [1] and Power-Efficient Gathering in Sensor Information System (PEGASIS) [2] assume sensor networks are homogeneous networks and does not perform well in heterogeneous networks, whereas, Stable Election Protocol (SEP) [3] and Distributed Energy- Efficient Clustering (DEEC) [4] deal with heterogeneous networks.

Distributed Energy Efficient Clustering (DEEC) is clustering - based algorithm in which cluster head is selected on the basis of probability of ratio of residual energy and average energy of the network. The round number of the routing epoch for each node is different according to its initial and residual energy. In this algorithm, the nodes with high initial and residual energy will have more chances to be the cluster heads compared to the low-energy nodes. As a result DEEC can prolong the network lifetime. But this algorithm penalizes the advanced nodes, when their residual energy deplete and become in the range of normal nodes. Because of this, the advanced nodes die quickly than the others.

In this paper, the proposed Modified Distributed Energy Efficient Clustering (MDEEC) protocol is evaluated by considering alive nodes for network lifetime, packet transmission to base station, energy consumption by comparing with LEACH and DEEC protocols.

\section{BACKGROUND}

Clustering procedures are engaged in dealing with energy control, where nodes are organized into cluster that communicate with a local base station and these local base stations transmit the data to the global base station, where it is accessed by the end user. This greatly reduces the distance, nodes need to transmit their data, as the local base station is close to all the nodes in the cluster.

\subsection{LEACH}

LEACH is a self-organizing, adaptive clustering protocol that uses randomization to distribute the energy load evenly among the sensors in the network. The sensor nodes organize themselves into local clusters, with one sensor node acting as the cluster head $(\mathrm{CH})$.The sensor nodes sense the data, transmit it to their associated $\mathrm{CHs}$ which receive, aggregate and then convey this data to the sink or to the Base Station (BS). All sensor nodes deployed in the field are homogeneous and have limited amount of energy. In order to divide the burden among the sensor nodes and also to improve network life time, clusters are formed. Nodes are made to become $\mathrm{CHs}$ on turns. Nodes randomly elect themselves as $\mathrm{CHs}$ and it is done in a way that each node becomes $\mathrm{CH}$ once in an epoch $1 / \mathrm{p}$. CHs selection is done on probabilistic basis, each node generates a random number $r$ inclusive of 0 and 1 , if generated value is less than threshold value given in the formula (i), then this sensor node becomes $\mathrm{CH}$.

$\mathrm{T}_{\mathrm{n}}= \begin{cases}\frac{\mathrm{p}}{1-\mathrm{p}\left[\mathrm{r} \cdot \bmod \frac{1}{\mathrm{p}}\right]} & \text { if } \mathrm{n} \in \mathrm{G}^{\prime} \\ 0 & \text { otherwise }\end{cases}$ 
Once clusters are formed, each $\mathrm{CH}$ broadcasts a TDMA schedule for sensor nodes associated with it. Nodes sense and transmit data to associated $\mathrm{CHs}$ during time slots assigned to them. Once each node in a cluster sent data, frame is repeated. In WSNs, main purpose is to control energy consumption and hence to increase network life. However, LEACH is not useful to be used in large areas due to energy constraint. The drawback of LEACH is that, once attributes are selected, they cannot be changed.

\subsection{DEEC}

DEEC is designed to deal with nodes of heterogeneous WSNs. For $\mathrm{CH}$ selection, DEEC uses initial and residual energy level of sensor nodes. Let $n_{i}$ denote the number of rounds to be a $\mathrm{CH}$ for sensor node $\mathrm{s}_{\mathrm{i}} \cdot \mathrm{p}_{\mathrm{opt}} \mathrm{N}$ is the optimum number of $\mathrm{CHs}$ in the network during each round. $\mathrm{CH}$ selection criteria in DEEC is based on energy level of nodes. As in homogenous network, when nodes have same amount of energy during each epoch then choosing $p_{i}=p_{o p t}$ assures that $\mathrm{p}_{\text {opt }} \mathrm{N}$ CHs during each round. In WSNs, sensor nodes with high energy are more probable to become $\mathrm{CH}$ than sensor nodes with low energy but the net value of $\mathrm{CHs}$ during each round is equal to $\mathrm{p}_{\mathrm{opt}} \mathrm{N}$. pi is the probability for each node $\mathrm{s}_{\mathrm{i}}$ to become $\mathrm{CH}$, so, sensor node with high energy has larger value of $p_{i}$ as compared to the $p_{\text {opt }} \cdot \bar{E}(r)$ denotes average energy of network during round $r$ which can be given as in [4]

$\bar{E}(r)=\frac{1}{N} \sum_{i=1}^{N} E_{i}(r)$

Probability for $\mathrm{CH}$ selection in DEEC is given as in [4 ]

$p_{i}=p_{o p t}\left[1-\frac{\bar{E}(r)-E_{i}(r)}{\bar{E}(r)}\right]=p_{o p t} \frac{E_{i}(r)}{\bar{E}(r)}$

In DEEC the average total number of $\mathrm{CH}$ during each round is given as in [4] :

$\sum_{i=1}^{N} p_{i}=\sum_{i=1}^{N} p_{\text {opt }} \frac{E_{\mathrm{i}}(\mathrm{r})}{\overline{\mathrm{E}(\mathrm{r})}}=p_{\text {opt }} \sum_{i=1}^{N} \frac{E_{i}(r)}{\bar{E}(r)}=N p_{\text {opt }}$

$\mathrm{p}_{\mathrm{i}}$ is probability of each sensor node to become $\mathrm{CH}$ in a round. Where $\mathrm{G}$ is the set of sensor nodes eligible to become $\mathrm{CH}$ at round $\mathrm{r}$. If sensor node becomes $\mathrm{CH}$ in recent rounds then it belongs to G. During each round each sensor node chooses a random number between 0 and 1 . If number is less than threshold as defined in equation below, it is eligible to become a $\mathrm{CH}$ else not.

$\mathrm{T}\left(\mathrm{s}_{\mathrm{i}}\right)=\left\{\begin{array}{cc}\frac{\mathrm{p}_{\mathrm{i}}}{1-\mathrm{p}_{\mathrm{i}}\left[\mathrm{r} \bmod \frac{1}{\mathrm{p}_{\mathrm{i}}}\right]} & \text { if } \mathrm{s}_{\mathrm{i}} \in \mathrm{G} \\ 0 & \text { otherwise }\end{array}\right.$

As $\mathrm{p}_{\mathrm{opt}}$ is reference value of average probability $\mathrm{p}_{\mathrm{i}}$. In homogenous networks, all sensor nodes have same initial energy so they use $p_{o p t}$ to be the reference energy for probability $\mathrm{p}_{\mathrm{i}}$. However in heterogeneous networks, the value of $\mathrm{p}_{\text {opt }}$ is different according to the initial energy of the sensor node. In two level heterogeneous network the value of $p_{o p t}$ is given by as in [4]:

$\mathrm{p}_{\mathrm{adv}}=\frac{\mathrm{p}_{\mathrm{opt}}}{(1+\mathrm{am})}$

$\mathrm{p}_{\mathrm{nrm}}=\frac{\mathrm{p}_{\mathrm{opt}}(1+\mathrm{a})}{(1+\mathrm{am})}$

Then use the above $\mathrm{p}_{\text {adv }}$ and $\mathrm{p}_{\text {nrm }}$ instead of $\mathrm{p}_{\mathrm{opt}}$ in equation 3 for two level heterogeneous network as supposed in [4]: $p_{i}=\left\{\begin{array}{cl}\frac{p_{\text {opt }} E_{i}(r)}{(1+a m) E(r)} & \text { if } s_{i} \text { is the normal node } \\ \frac{p_{\text {opt }}(1+a) E_{i}(r)}{(1+a m) E(r)} & \text { if } s_{i} \text { is the advanced node }\end{array}\right.$

In DEEC, average energy $\mathrm{E}(\mathrm{r})$ of the network for any round $\mathrm{r}$ has been estimated as in [4]:

$\bar{E}(r)=\frac{1}{N} E_{\text {total }}\left(1-\frac{r}{R}\right)$

$\mathrm{R}$ denotes total rounds of network lifetime and is estimated as follows:

$R=\frac{E_{\text {total }}}{E_{\text {round }}}$

$E_{\text {total }}$ is total energy of the network where $E_{\text {round }}$ is energy expenditure during each round

\section{PROPOSED PROTOCOL}

The proposed protocol implements the same concept as in DEEC in terms of selecting $\mathrm{CH}$ which is based on residual energy level of the sensor nodes with respect to average energy of the network. In DEEC it can be seen that the sensor nodes with more residual energy at round $\mathrm{r}$ are more probable to become $\mathrm{CH}$, so, in this way sensor nodes having higher energy values or advanced nodes will become $\mathrm{CH}$ more often as compared to the sensor nodes with lower energy or normal nodes. A situation arises in a network where advanced nodes having same residual energy like normal nodes. After this point DEEC continues to punish the advanced nodes so this is not optimal way for energy distribution because by doing so, advanced nodes are continuously a $\mathrm{CH}$ and they die more quickly than normal nodes.

\section{Setup Phase}

The proposed protocol uses same mechanism for $\mathrm{CH}$ selection and average energy estimation as proposed in DEEC. At each round, sensor nodes decide whether to become a $\mathrm{CH}$ or not by choosing a random number between 0 and 1 . If number is less than threshold $\mathrm{T}_{\mathrm{s}}$ as shown in equation (x) then sensor nodes decide to become a $\mathrm{CH}$ for the given round. In this, threshold value is adjusted and based upon that value a sensor node decides whether to become a $\mathrm{CH}$ or not by introducing residual energy and average energy of that round with respect to optimum number of $\mathrm{CHs}$.

Threshold value is given as

$\mathrm{T}(\mathrm{s})=\left\{\begin{array}{c}\frac{\mathrm{p}}{1-\mathrm{p}\left(\mathrm{r} \bmod \frac{1}{\mathrm{p}}\right)} * \frac{\text { Residual Energy of a node } * \mathrm{k}_{\mathrm{opt}}}{\text { Average energy of network }} \\ 0 \quad \text { otherwise }\end{array}\right.$ if $\mathrm{s} \in \mathrm{G}$

The cluster head sets up a TDMA schedule and transmits it to the sensor nodes in the cluster. After the TDMA schedule is known by all the sensor nodes in the cluster, the setup phase is completed and the next phase begins.

\section{Steady state phase}

Once the clusters are established, the sensor nodes transmit their data messages towards the cluster-head. In this phase, before communication, the proposed algorithm checks whether the sensor node is cluster-head or a cluster member. This helps the $\mathrm{CH}$ in preventing to pass the packet to itself. Thus cluster-head communicates with its cluster 
members and BS only. Within the cluster, the communication uses TDMA, as described in the set up phase. When the cluster-head receives all the nodes data, it performs its compression, to form a new message that sent to the base station.

\section{SIMULATION AND RESULTS}

In this section, LEACH, DEEC and proposed routing protocol MDEEC for wireless sensor network are simulated in MATLAB environment. For simulation, a network consisting of 100 nodes randomly placed in a field of dimension $100 \mathrm{~m} \mathrm{x}$ $100 \mathrm{~m}$ and a BS located in the center is considered. For simplicity, all nodes are considered to be fixed and energy loss due to signal collision and interference between signals of different nodes that are due to dynamic random channel conditions is ignored.

\section{Simulation parameters}

\begin{tabular}{|c|c|}
\hline Parameters & Values \\
\hline Network field & $100 \mathrm{~m}, 100 \mathrm{~m}$ \\
\hline Number of nodes & 100 \\
\hline Eo(initial energy of normal nodes) & $0.5 \mathrm{~J}$ \\
\hline Message size & $4000 \mathrm{bits}$ \\
\hline $\mathrm{E}_{\text {elec }}$ & $50 \mathrm{~nJ} / \mathrm{bit}$ \\
\hline $\mathrm{E}_{\mathrm{fs}}$ & $10 \mathrm{~nJ} / \mathrm{bit} / \mathrm{m} 2$ \\
\hline $\mathrm{E}_{\mathrm{amp}}$ & $0.0013 \mathrm{pJ} / \mathrm{bit} / \mathrm{m} 4$ \\
\hline $\mathrm{E}_{\mathrm{DA}}$ & $5 \mathrm{~nJ} / \mathrm{bit} / \mathrm{signal}$ \\
\hline $\mathrm{d}_{\mathrm{o}}$ (threshold distance) & $70 \mathrm{~m}$ \\
\hline $\mathrm{P}_{\mathrm{opt}}$ & 0.1 \\
\hline
\end{tabular}

Figure 1 shows the comparison of protocols LEACH, DEEC and MDEEC considering dead nodes relative to the number of rounds. From figure 1, it can be seen that the first node for LEACH, DEEC and MDEEC dies at 1040, 1454 and 1398 rounds respectively. Tenth node dies at 1080, 1631 and 1580 rounds respectively and all the nodes are dead at 1547, 3443 and 3971 rounds respectively. Therefore, from the simulated results of these three protocols, it can be concluded that in terms of stability period, MDEEC performs better.

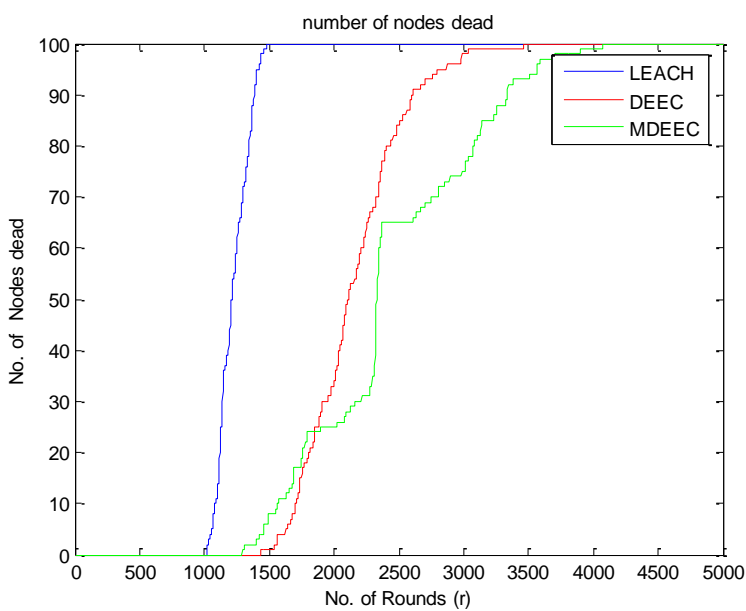

Figure 1. Nodes dead during rounds

In figure 2, graph shows that number of alive nodes during each transmission round for the LEACH, DEEC and MDEEC routing protocol. From the graph, it can be seen that network life time is enhanced quite significantly when compared with other approaches, that is when compared with LEACH and DEEC, MDEEC performs better. Nodes alive up to rounds $\mathrm{r}=$ 1547 in LEACH, $\mathrm{r}=3443$ in DEEC and in MDEEC nodes remain alive up to 3971 rounds. Thus this shows that in the case of MDEEC the nodes remain alive for longer time than LEACH and DEEC routing protocols.

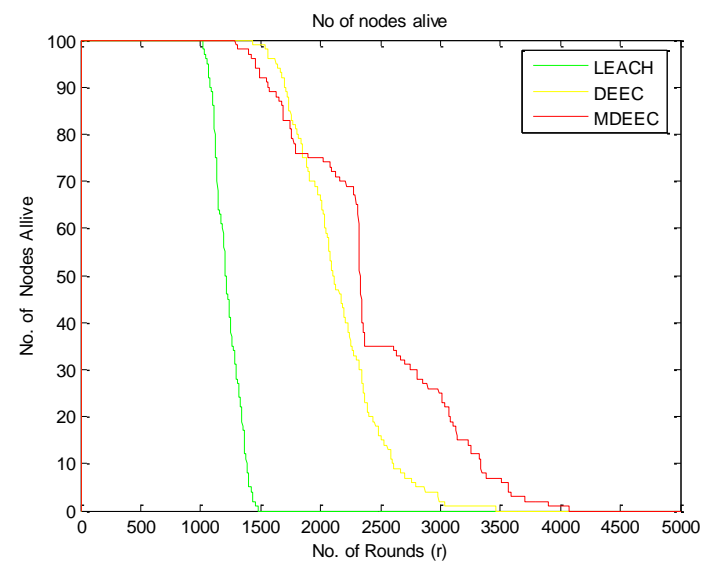

Figure 2. Nodes alive during rounds

Figure 3 shows that total initial energy of the network which decreases linearly up to 1500 rounds and after that there is a difference from the round where the first node dies in the case of LEACH, DEEC and MDEEC. From the graph it can be clearly seen that the energy remaining per round for MDEEC is more as compared to LEACH and DEEC.

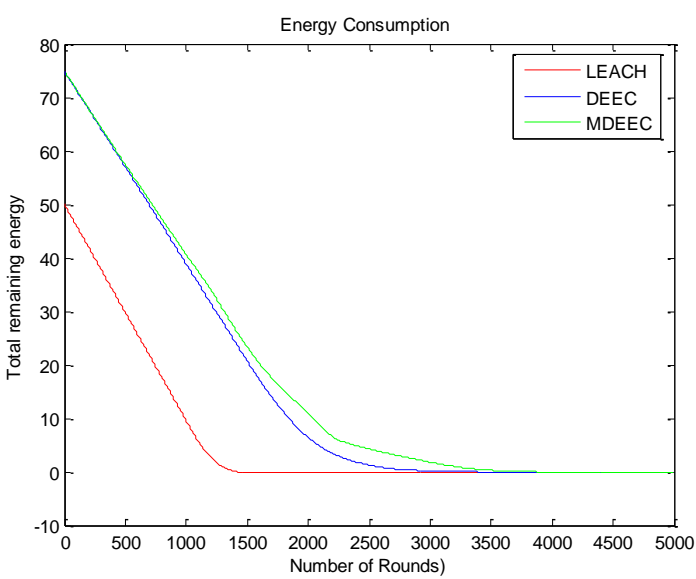

Figure 3: Total remaining energy over rounds

In Figure 4, number of data packets to BS is calculated for all routing protocols that is, for LEACH, DEEC and MDEEC. It shows that MDEEC is efficient in successful data delivery. In other words throughput increases quiet remarkably. 


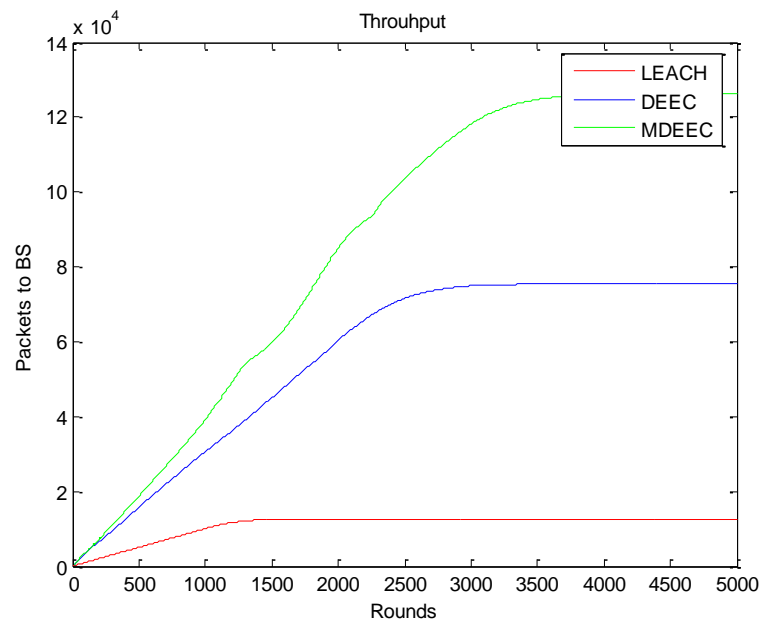

Figure 4: Number of Data Packets sent from CHs to BS

Figure 5 shows the $\mathrm{CHs}$ selection of the three routing protocols that is LEACH, DEEC and MDEEC, and it can be seen that, MDEEC provides optimal number of $\mathrm{CHs}$ for every round.
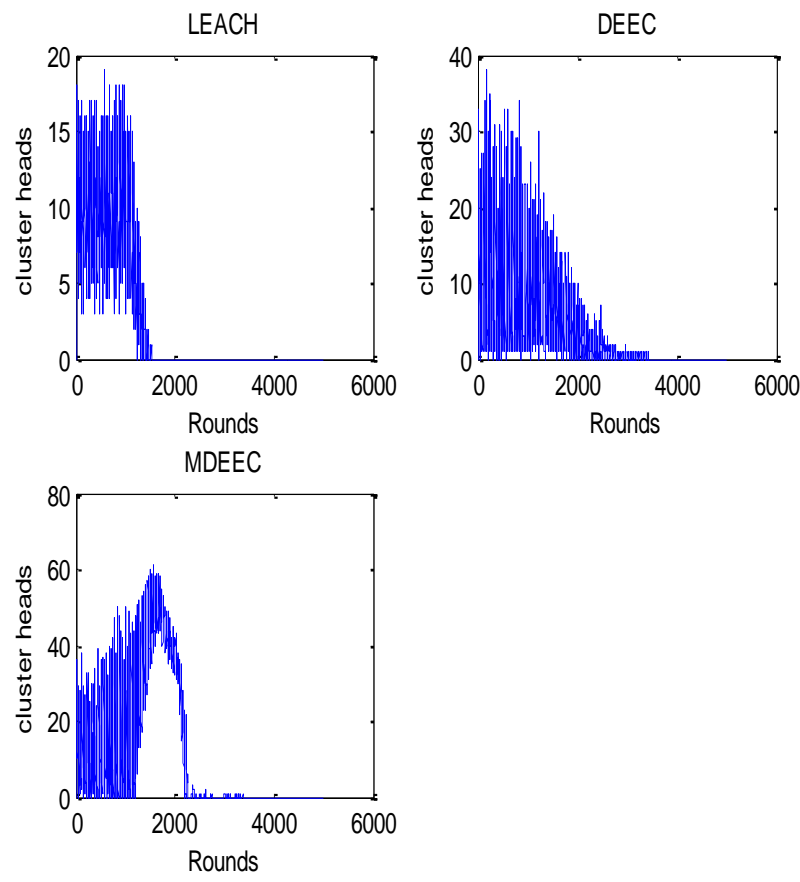

Figure 5: Cluster heads per round

The figure 6 presents for different values of initial energy, all nodes died round. The results of the figure 6 prove that for different values of initial energy $\mathrm{E}_{0}$, MDEEC performs better as its all nodes died round is always larger than the $\mathrm{LEACH}$ and DEEC ones.

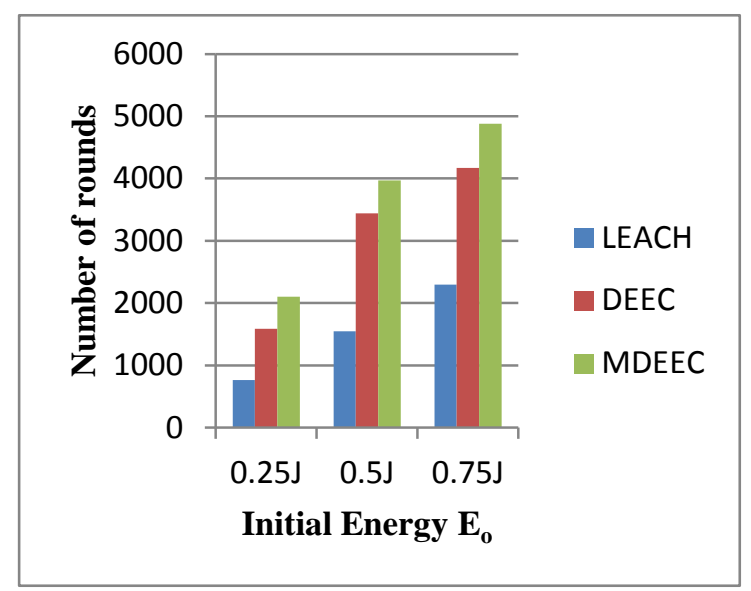

Figure 6: Performance results for all nodes die with different values of $E_{0}$

The figure 7 presents for different values of initial energy, number of packets sent from $\mathrm{CHs}$ to base station.. The results of the figure 7 prove that for different values of initial energy $\mathrm{E}_{0}$, MDEEC performs better as the number of packets sent from $\mathrm{CHs}$ to $\mathrm{BS}$ is always larger than the LEACH and DEEC routing protocols

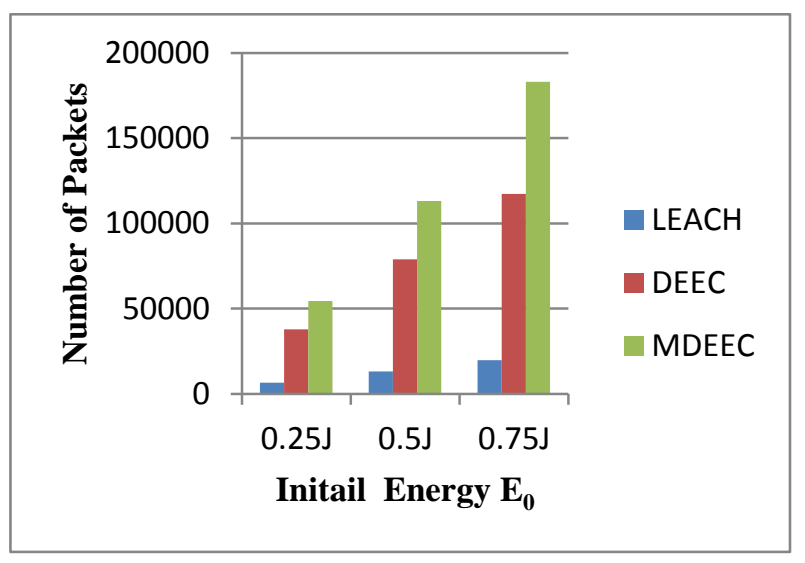

Figure 7: Performance results for number of packets sent from CHs to BS with different values of $E_{0}$

\section{CONCLUSION}

In this paper, Modified Distributed Energy Efficient Clustering Routing Protocol (MDEEC), an energy-aware adaptive clustering protocol used in heterogeneous wireless sensor networks has been proposed, examined and compared with LEACH and DEEC routing protocols. The proposed algorithm shows better performance in terms of energy saving, alive nodes and packet transmission. Simulation results shows that MDEEC has better performance as compared to LEACH and DEEC.

\section{ACKNOWLEDGMENT}

The authors would like to thank UGC for providing fund for the project with an entitled "Design Tool of IPv6 Mobility for 4G-Networks", under eleventh plan of Major Research Project scheme (Ref.No F.No 36- 167/2008(SR) dated 26.03.2009). Thanks also goes to the dedicated research group in the area of Wireless Sensor Networking, Cloud \& Grid Computing and e-CRM at the Dept of Computer 
Science, Mangalore University, Mangalore, India, for many stimulating discussions for further improvement and future aspects of the project. Lastly but not least the author would like to thank everyone, including the anonymous reviewers.

\section{REFERENCES}

[1] W.Heinzelman, A. Chandrakasan, and $H$. Balakrishnan, "Energy-efficient communication protocol for wireless sensor networks," in the Proceeding of the Hawaii International Conference System Sciences, Hawaii, January 2000

[2] S. Lindsey and C. S. Raghavendra, "PEGASIS: Power Efficient GAthering in Sensor Information Systems," in the Proceedings of the IEEE Aerospace Conference, Big Sky, Montana, March 2002.

[3] G. Smaragdakis, I. Matta, A. Bestavros, "SEP: A Stable Election Protocol for clustered heterogeneous wireless sensor networks." in: Second International Workshop on Sensor and Actor Network Protocols and Applications (SANPA 2004), 2004.

[4] L. Qing, Q. Zhu, M. Wang, "Design of a distributed energy- efficient clustering algorithm for heterogeneous wireless sensor networks". ELSEVIER, Computer Communications 29, pp 2230- 2237, 2006.
[5] Jamal N. Al-Karaki, Ahmed E. Kamal, "Routing Techniques in Wireless Sensor Networks: A Survey", IEEE Wireless Communications, Volume: 11, Issue: 6, 26-28, December 2004

[6] A. Manjeshwar and D. P. Agarwal, "TEEN: a routing protocol for enhanced efficiency in wireless sensor networks," In 1st International Workshop on Parallel and Distributed Computing Issues in Wireless Networks and Mobile Computing, April 2001

[7] A. Manjeshwar and D. P. Agarwal, "APTEEN: A hybrid protocol for efficient routing and comprehensive information retrieval in wireless sensor networks," Parallel and Distributed Processing Symposium., Proceedings International, IPDPS 2002, pp. 195-202

[8] U. Sajjanhar, P. Mitra, "Distributive Energy Efficient Adaptive Clustering Protocol for Wireless Sensor Networks" Proceedings of the 2007 International Conference on Mobile Data Management, pp. 326 330, 2007.

[9] Ma Chaw Mon Thein, Thandar Thein "An Energy Efficient Cluster-Head Selection for Wireless Sensor Networks", International Conference on Intelligent Systems, Modeling and Simulation, IEEE 2009. 